\title{
Group-based Assessments and Project Management Education: Towards a Dynamic Framework with Best Practices
}

\author{
Roksana Jahan Tumpa ${ }^{1}$, Samer Skaik ${ }^{2}$, Ghulam Chaudhry ${ }^{3}$, Miriam Ham ${ }^{4}$ \\ Central Queensland University, Sydney, $N S W^{l}$, Central Queensland University, \\ Melbourne, VIC ${ }^{2}$, Central Queensland University, Sydney, NSW ${ }^{3}$, Central Queensland \\ University, Cairns, $Q L D^{4}$ \\ Australia
}

\begin{abstract}
The skill sets required for the successful completion of projects have changed dramatically in recent years. Employers now expect project practitioners to possess a balanced mixture of soft and technical skills. However, employers are often dissatisfied that project management graduates are not equipped with the right set of skills. The use of group-based assessments in higher education institutions (HEIs) is becoming increasingly important to develop the demanded project management competencies. The study aims to develop a framework for designing and administering groupbased assessments through a systematic literature review (SLR). The proposed framework is then to be validated to improve its effectiveness and rigour through data gathered from interviews and focus groups. It is expected that the higher education sector will benefit from the outcomes of the research as the validated framework will guide academics to design group-based assessments rigorously and systematically. The paper discusses the relevant literature, the gaps in the literature, the research aims, questions, method, discussion, and the implications of the findings.
\end{abstract}

\section{Introduction}

The project environment is a dynamic system. In the dynamic environment, project managers are required to perform through the uncertainty and complexity of the project environment. As a result, they require skills to quickly adapt to situations that are within and outside of their control [5].

Employers now require graduates to possess a mixture of soft skills, disciplinary technical skills and project management hard skills [2]. Soft skills include communication skills, teamwork, problem-solving, creativity, interpersonal skills, critical thinking, and leadership traits. On the other hand, disciplinary technical skills refer to industry-specific skills. For instance, for a construction project manager job, it is desirable to employ a project manager with a civil engineering degree. Project management hard skills include management of time, cost and quality within a project [2].

Employers are often dissatisfied that project management (PM) graduates are not well equipped with the right set of skills and often demonstrate a lack of soft skills in particular [5]. Employers often claim that universities do not place sufficient emphasis on the development of soft skills. In order to respond to employers' demands, considerable efforts have been made to develop graduate employability by teaching and developing soft and technical skills. In the extant literature, there are many approaches available to developing project management competencies in graduates [5], the research focuses on the function of group-based assessments to cultivate PM competencies in graduates. The study proposes a framework to inform the design and administration of group-based assessments in higher education to enhance employability and readiness to join the workforce.

\section{Group-based assessments}

Group-based refers to the necessity of a collaborative group environment in which the assessment task is undertaken [6]. In order to respond to employers' demands and enhance employability of project management graduates, group-based assessments have been introduced as a pedagogical tool in higher education in recent years.

Projects require the engagement of teams across geographical regions, commitment, and participation to deliver the projects within budget and time [7]. As projects involve the collaboration of numerous stakeholders, project management graduates are expected of working collaboratively in a team [4]. As a result, several disciplines have incorporated group- 
based assessments to develop team-building skills and promote deep learning in graduates [6].

Group-based assessments have long been used in higher education across disciplines in order to respond to employers' demand and development of soft and technical skills in graduates [5]. The assessments seem to be improving students' soft skills such as interpersonal skills, team building, leadership and communication skills [6]. In collaborative learning, students achieve better outcomes, obtain deep learning, acquire greater communication and teamwork skills, and gain a better understanding of the environment in which they will be working as professionals.

Despite numerous benefits, educators and students experience challenges while working on a groupbased assessment. Free-riding and social loafing are the most frequently cited issues in the literature [4]. However, academics can alleviate these issues through careful planning of group-based assessments.

\section{Good practices of group-based assessments}

\subsection{Course considerations}

Teachers should consider whether group assessments are suitable or not before group-based assessments are included in a course. Excessive use of group-based assessments limits students' ability to think, learn, work independently and creates "group fatigue" thus developing coping strategies including cognitive shortcuts [9]. Academics believed that the weighting, frequency and timing of group-based assessments should be considered in a whole-ofcourse approach. It is recommended to keep a group assessment's weightage to $30 \%$ both in each subject and in a course [3].

The initial literature review postulates that there is a paucity of empirical research about the number and timing of group-based assessments in a course. Thus, a thorough investigation is required around this aspect to investigate whether programme consideration should be recognised while designing and administering group-based assessments in the curriculum.

\subsection{Prior instructions}

Students are required to be trained in group work skills and should be provided with teamwork knowledge, skills, and abilities before they are involved in group-based assessments [14]. Training students in teamwork can lead to higher academic achievements [14]. If the development of teamwork skills is a learning outcome in a course or program of study, then relevant training and instruction in teamwork must be incorporated into its design at the planning phase. [13] conducted a SLR on the factors that afford teamwork pedagogy in higher education. They found that educators often place students in teams with little or no instruction on how to work in teams before assigning complex team projects.

\subsection{Group formation}

The three most frequently used group formation processes found in the literature are student-selected, the selection made by instructors [11], and randomly selected groups [10].

Student-selected groups are generally homogeneous resulting in members establishing better communication, getting along with each other and demonstrating enthusiasm towards working efficiently than members of randomly assigned groups [10]. [20] demonstrated that student-selected groups have the potential to reduce students' complaints about team structures. Contrarily, studentselected group formation was discouraged as close friends seem to be deceitful in the context of education and learning in the team and they were less taskoriented [12]. The outcomes of the assessment tended to be pessimistic as the quality of the work was compromised at the expense of exchanging mutual benefits [12].

Considering the drawbacks of student-selected groups, teachers should use discretion when forming groups [11]. Students in instructor-driven groups reported better learning than self-selected groups [1]. However, if the groups are formed in accordance with teachers' choice, it may lead to continuous student objections, thus creating an uncomfortable group environment and reducing group performance [20].

Random group formation method encompasses teachers forming groups with the random selection of students. Once teachers construct groups randomly, students seem to be dissatisfied with the team structures. In the random group formation process, within the time constraints, teachers may decide to form groups randomly [20]. On the other hand, the results of carefully engineered groups (balanced) and random groups showed that there was no significant difference in outcomes between balanced and randomly selected groups [10]. The results of the study were in accordance with the findings of [4]. They demonstrated that there is no correlation between the group formation process and the decrease in the tendency of a social loafing attitude.

Taken together, it is plausible to anticipate that the literature suggests that although group formation is a design feature of group-based assessments, there is a disparity in pedagogical implementation of group 
formation techniques among academics. Hence, this research will identify the best practices of group formation methods by employing a SLR based on evidence-based scholarly studies.

\subsection{Group size}

The existing literature around group size revealed contention in opinion among researchers. However, a smaller group size was preferred in the extant literature [4]. Larger group size stimulates social loafing attitude in students because it is easy for social loafers to hide in the large group.

Smaller groups generally perform better than larger teams. The finding has been obtained in the study of [4], [6], [18]. In these studies, small group size was preferred to reduce social loafing and freeriding issues. On the other hand, group size was not related to self-rated learning. A group with five to seven students were generally preferred, but group tasks need to be considered before determining group size [1].

It is apparent from the discussion that achieving a conclusive finding regarding the group size in groupbased assessments is far from settled. Academics differ in their opinion about the exact number of team members and its pedagogical implementation in the curriculum, although smaller group size is recommended. As a result, the gap calls for a SLR to identify the best practices regarding group size.

\subsection{Group member evaluation}

In order to reduce free-riding issues in groupbased assessments and to better appraise an individual's contribution, self and peer evaluations are a prevalent tool in group-based assessments [17]. Self and peer assessments manifested a plethora of advantages which include engaging in developing critical thinking, improving team-building skills, and cultivating interpersonal attributes in students [18].

The employment of group member evaluations to moderate a group score or grade into individual scores has been outlined in many studies [8]. One of the common techniques to extract individual scores is the Self and Peer Assessment Resource Kit (SPARK) [8]. Recent advancement in computer technology has made the interaction easier among group members enabling them in sharing their files and logs [16].

The grading structure of group-based assessments should be based on the team learning model performance evaluation system [16]. As per this model, an individual's grade in a group assessment should be based on individual performance, group performance, and peer evaluation. A small percentage of the grade $(10 \%)$ taken from the group member evaluation prompted students to work more diligently. However, such grading structure sometimes is not approached appropriately by students while their marks are dependent on their group members.

Group member evaluation papers were introduced to discuss the evaluation and progress of the group [16]. The group member evaluation paper has two parts. In the first part, students need to explain what they learnt about the behaviour of the group from the textbook and lecturer. In the second part, students provide anonymous feedback to their group members which give teachers an opportunity to identify the presence of social loafers or free riders in the group [16].

Group member evaluations seem to hold great promise pertaining to students' engagement and interaction in collaborative learning. However, the evaluation process might have biases when it comes to assessing group members, thus raising questions about its reliability and validity. Students raised concerns about the fairness and accuracy of the group member evaluation [17]. Feedback provided by a nonexpert person is also dubious due to its quality, reliability and the degree of student's understanding about the expectation of work [17].

It can be concluded that self and peer evaluations in a group assessment are valuable for developing an array of such attributes as team building, problemsolving and transferable skills. Nevertheless, self and peer assessments involve some challenges. A comprehensive literature review regarding self and peer evaluations through a SLR is expected to bring some insights about dealing with the challenges.

\section{Identified knowledge gaps}

A scoping literature review was conducted to verify whether there are SLRs conducted on the best practices of group-based assessments in the context of project management. Some systematic literature reviews are already available in group-based assessments in the context of higher education; however, none was found which systematically accumulates best practices for designing and administering group-based assessments. For instance, [7] explored and synthesised the studies about groupbased assessments in educational settings and found that social skills development, peer assessment, and group processes have become the focus of groupbased assessments instead of the assessment of knowledge acquisition. On the other hand, [13] investigated the factors perceived to afford or constrain teamwork pedagogy in higher education. The research focused merely on the pedagogical aspects of teamwork. In addition, the review did not provide any best practice advice on the design features 
of group-based assessments. In addition, the inclusion criteria of the review did not mention whether the literature included in the review was based on groupbased assessments.

The existing literature suggests that there are several good practices of group-based assessments and strategies to reduce the challenges of group work. In addition, some studies integrated the good practices of group-based assessments [6]. Nevertheless, those studies did not systematically evaluate the best practices for group-based assessments. The available literature lacks consensus as to how to integrate all the best practices of group-based assessment. It is suggested that the findings of the SLR will inform this integration.

Considering group-based assessments are a widespread tool to develop PM competencies in graduates, there is a dearth of research which systematically considers all best practices, particularly in the area of project management. It is hoped that the development of a framework for groupbased assessments will enhance the benefits, reduce the drawbacks and address the clear gaps which need attention.

\section{Research aims and Objectives}

The research conducted aims to propose a framework for designing and administering groupbased assessments to foster competencies of PM graduates. The objectives of this research are to (1) identify the best practices for conducting group-based assessments, (2) develop a framework for groupbased assessments, and (3) validate the proposed framework for improving its effectiveness and rigour.

\section{Research questions}

Considering the importance of cultivating PM competencies in graduates and group-based assessments being a common practice in higher education, this research aims to answer the following research questions:

RQ 1: What are the best practices of group-based assessments that aim to develop project management competencies of higher education graduates?

RQ 2: What are the perceptions of project management academics regarding the emergent best practices of group-based assessments?

RQ 3: What constitutes a framework integrating project management academics' perceptions about the best practices and their practices of conducting groupbased assessments?

\section{Research method}

In order to achieve the aims and objectives of the research, data collection will be conducted in three stages (see Figure 1). The stages of this research are described below:

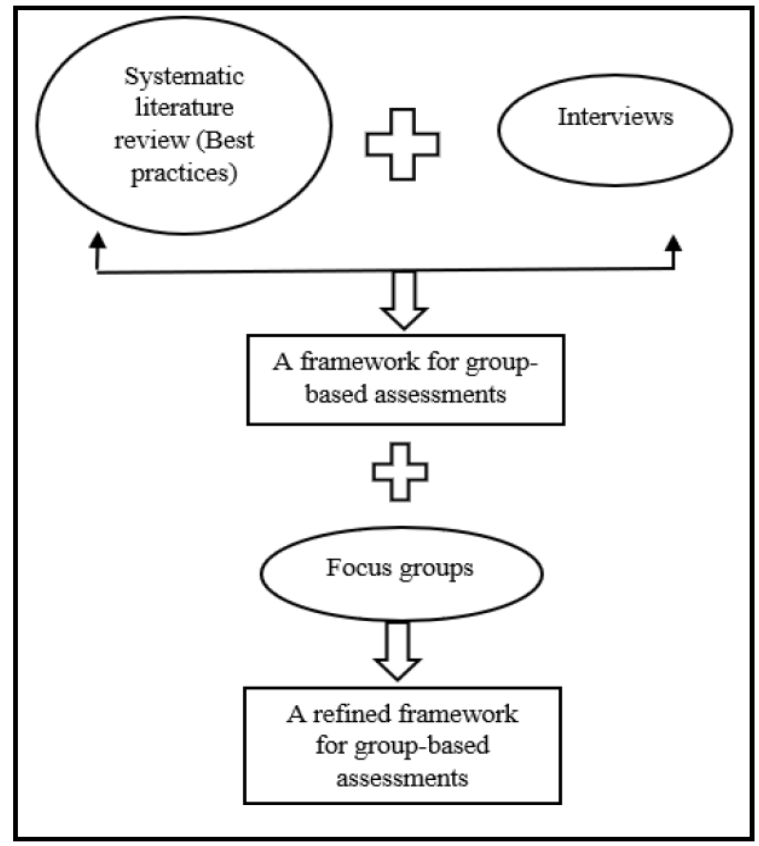

Figure 1. Stages of the research

\subsection{First stage - systematic literature review}

In the first stage, a SLR will be conducted to collect evidence-based best practices in group-based assessments. A systematic literature review considers all available studies within the set inclusion and exclusion criteria, and as a result, this methodology seems to be transparent in comparison to narrative reviews [15]. The results or data synthesis are based on a high-quality investigation in systematic literature reviews. In the systematic literature, evidence-based data will be collected from the existing literature and built on by providing a new way of looking at the role of group-based assessments in the development of PM competencies in graduates.

The review will be adhering to the Preferred Reporting Items for Systematic Reviews and MetaAnalyses (PRISMA). The steps involved as per the PRISMA checklist are described below.

7.1.1 Scoping. A great deal of scholarly work that describes group formation techniques, challenges of group-based assessments, group composition techniques, group size, task complexity, team charter and self and peer assessments was evident in the 
existent literature. The existing literature lacks research which systematically reviews all the variables for designing and administering groupbased assessments. To address the aims and objectives of this research project, a research question has been formulated (RQ 1).

7.1.2 Planning. The key search terms and their synonyms will be identified based on the research question (RQ 1). The keywords are group-based assessments, group projects, group-based assignments, group assessments, group assignments, team projects, team-based assignments, team assessments, team assignments.

7.1.3. Identification. The database search will be conducted in this phase. The keywords will be applied in different databases to identify primary studies. The databases for searching group-based assessments will be A+ Education, Education Research Complete, Emerald, ERIC, Taylor and Francis, SAGE, ScienceDirect, ProQuest One Academic, Gale Academic Onefile, and Teachers Reference Centre (see Figure 2).

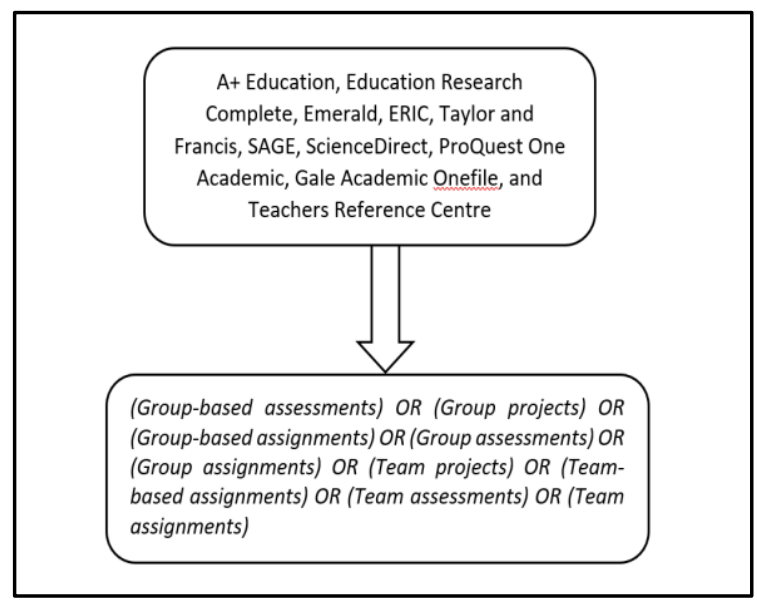

Figure 2. Library database search

7.1.4 Screening. The search results identified in different databases will be imported to a Microsoft Excel sheet to remove the duplicates across databases. The inclusion criteria include:

- (Described in English) AND

- (Peer-reviewed journal articles) AND

- (Full text only) AND

- In the year range $(2010$ - 2020) AND

- (Articles based on empirical studies)

The exclusion criteria include:

- Any study related to primary school, secondary education, vocational education and training, and workplace sectors
- Conference proceedings

- Reports based on group-based assessments

- Book chapters

- Dissertations

Once duplicates are removed, the inclusion and exclusion criteria will be applied to the title and abstract of the selected scholarly studies. If the title and abstract meet the inclusion and exclusion criteria, then the full text of the potentially eligible studies will be obtained. If the title and abstract do not meet the inclusion and exclusion criteria, the study will be rejected.

7.1.5 Eligibility. If the title and abstract meet the inclusion and exclusion criteria, the article will be included in the list of eligible studies. The relevancy of potentially eligible studies will be checked in this phase by thoroughly reading the full text to make sure it is relevant. In the full-text screening, the focus will be on the result, discussion and conclusion sections. The studies which are not closely related to the research question (RQ 1) will be rejected at this stage. Any rejected articles will be tabulated with a reason for rejection to increase the transparency of the selection.

7.1.6 Inclusion of information. In this stage, the selected studies will be read carefully, and all relevant information related to the research question (RQ 1) will be extracted for inclusion from the result, discussion and conclusion sections of the retained articles. Data will be extracted manually related to population, intervention, comparative intervention, and outcomes of the included studies. To maintain consistency and minimise bias in the data extraction throughout the selected studies, a separate data extraction form for qualitative and quantitative studies will be complied.

Data extraction should be performed independently by two or more researchers whenever feasible [15]. However, when the researcher is the sole researcher, other checking techniques can be used. A test-retest process will be employed where primary data will be extracted randomly for a second time to confirm the consistency of the data extraction. After completing the data collection process at the outset of PRISMA, the following flow chart (see Figure 3) will be populated with the data collected from the findings of adhering to the PRISMA process. 


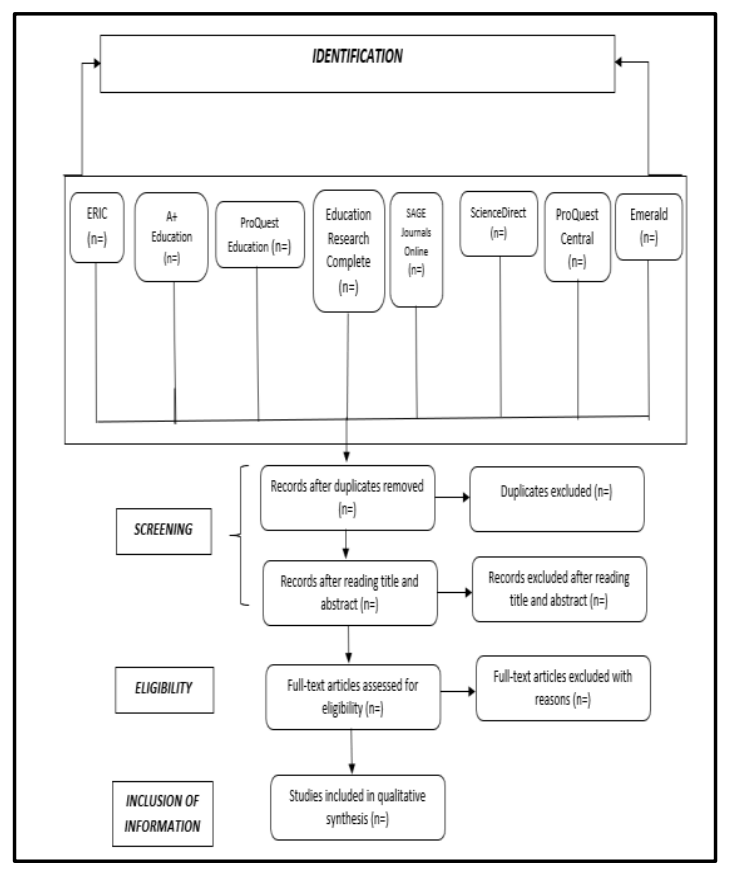

Figure 3. PRISMA flow chart for the systematic literature review

7.1.7 Data analysis of SLR. Data analysis involves the collation, combination and summary of the findings of individual studies included in the systematic literature review to draw a conclusion to answer the research question [15]. If the systematic literature review has both qualitative and quantitative studies, data synthesis should be done separately [15]. This is the approach that will be followed for this research.

Data can be analysed from the quantitative studies either narratively or statistically (meta-analysis) [15]. If the interventions, designs and outcomes are similar, it may be possible to conduct statistical analysis (meta-analysis). Otherwise, a narrative (descriptive) synthesis will be employed to answer the research question (RQ 1).

For the purpose of this research, a preliminary tabulation technique will be used to analyse the quantitative data because of the simplicity of its implementation. Tabulation is widely used for presenting quantitative data visually. Tabulation can be useful at any stage of the preliminary synthesis process; however, the researcher of this study will be using it in the data analysis stage. The tables will include the type of paper, methodological approach, participant information and inclusion criteria, study location, and summary of main study findings. The subject matter of the findings of the quantitative studies will be examined and placed under the qualitative themes accordingly.
The data extracted from qualitative studies can be analysed in a number of ways. A thematic analysis approach to analyse the data will be employed considering major themes. The steps involved in thematic analysis are familiarisation, coding, theme development, reviewing, defining themes and producing a report [19].

Familiarisation is a process of making the researcher familiar with the dataset which facilitates active engagement with the data [19]. Once, the relevant information is extracted from the selected articles, that information will be carefully read. Having developed a good sense of the extracted information, the specific segment of the dataset will be coded. This process is called generating codes [19].

Constructing themes from coded data will be carried out by combining, clustering or collapsing codes together into bigger or more meaningful patterns [19]. An inductive thematic approach will be utilised to generate themes from the raw data.

The identified themes will then be checked against the coded data, the dataset and the research question (RQ 1) to ensure the quality of the themes. In reviewing, some themes may be rejected. The final review is to check whether the story that has emerged through the identified themes answers the research question (RQ 1). Finally, a final report will be presented to answer research question (RQ 1) through identified themes.

\subsection{Second stage - interviews}

In the second phase, project management academics will be interviewed to collect information about their good practices in conducting group-based assessments. Interviewees will also be providing their opinion about the identified best practices derived from the SLR.

One-to-one interviews with academics will be carried out for the research. Since the interviewees need to provide their detailed opinion about the emergent best practices and share their practices of conducting group-based assessments, one-to-one interviews would be appropriate.

Structured interviews consist of a few questions, provide relatively short answers to the posed questions and limit the opportunity of providing elaboration on subject matters. Unstructured interviews encourage interviewees to talk around themes, issues or topics but generate extensive transcripts which may be difficult to compare and integrate for a novice researcher. For this study, the interviewees need to shed light on the best practices for administering group-based assessments. Hence, semi-structured interviews would be pertinent. This approach will allow the interviewees to answer the 
predefined questions set by the researcher but also an open-ended discussion about their practices.

A purposive sampling technique will ensure the right selection of the interviewees. Included participants will be full-time project management faculty members at an Australian university who currently conduct group-based assignments, and utilise a minimum of one group-based assessment in their course. The number of participants in the interview will be between 23-25.

The researcher aims to carry out face-to-face interviews, however, Skype or Zoom interviews will also be considered due to the COVID-19 pandemic. The duration of each interview will be approximately 60 to 90 minutes. The potential interviewees will provide their perceptions about the best practices and their pedagogy of group-based assessments. The conversation will be recorded using an audio recorder or Zoom recording settings. Extensive field notes will also be taken to augment the audio taping.

Integrating the academics' good practices in conducting group-based assessments and the best practices that emerged from the SLR, a framework will be proposed for the context of project management.

7.2.1 Data analysis of interviews. Interview recordings will be transcribed verbatim. The transcripts will be analysed using NVivo. Open coding will be utlised for the identification of themes or categories in the data and placing a preliminary label on them. The process will answer the second research question [RQ 2] about the perceptions of project management academics with regard to the emergent best practices of group-based assessments. It is expected that some themes will fit into those previously identified by the SLR. Inductive thematic coding will then be conducted to identify any new theme that emerged from academics about their practices of conducting group-based assessments. A list of refined themes will then emerge after comparing and contrasting the themes from the findings of the SLR and the academics' practices. The process aims to develop a framework for designing and administering group-based assessments which will answer the second question of the study [RQ 2].

\subsection{Stage 3 - focus groups}

The framework developed in the second stage of the research will be validated to improve its rigour, effectiveness and practicality using focus groups. To validate the framework, two focus groups will be conducted at two Australian universities.

In the focus groups, participants will be providing their opinion about the framework. The target population of the focus groups will be project management academics. However, they will be a different group than those who participated in the interview. The academics for the focus groups will be selected from two Australian universities. Each focus group will consist of six to eight academics.

A purposive sampling technique will be employed to allow the researcher to select the participants who can give appropriate information. The participants of the focus group will be selected based on their diverse experience and background in learning and teaching and their involvement in designing group-based assessments. The essential inclusion criteria are that academics have to be full-time academics teaching in higher education institutions in Australia with a position ranging from lecturer to professor. The focus group study will be limited to Australia only because of budget constraints. The focus groups, therefore, should be composed of those who are similar to each other. In this study, the similarity of focus group participants will be delineated as those who are faculty members designing and implementing groupbased assessments. The selected universities' staff directory will be searched for the prospective academics' name, positions, years of teaching experiences, phone numbers and email address. A potential participant list will be prepared depending on the positions of the academics. Then, a simple random sampling will be employed to avoid selection bias. The selected academics will be contacted via emails to seek their consent to participate in the study. Upon their supplying consent, the interviewees will be provided with the framework derived from the second stage of research.

The researcher will seek assistance from an experienced moderator in order to ensure the effectiveness of the focus groups. The focus group discussion will be kept between 60 to 120 minutes. In the focus group, the interviewees will be sharing their opinion about the framework. The conversation will be recorded using an audio recorder.

7.3.1 Data analysis of focus groups. The audio recording will be transcribed verbatim. The feedback from the focus group interviewees will be analysed thematically using Nvivo. Then the emergent themes will be compared and contrasted with the framework derived from the second stage. The agreement and disagreement between and within focus group participants regarding the framework will be critically analysed and reported.

\section{Discussion}

Group-based assessments have been discussed extensively in the literature in order to develop 
students' team building, critical thinking, problemsolving skills, communication skills and leadership traits. However, students experience many problems when it comes to working in a group assessment. Some issues include non-contributing peers, unfair distribution of grades, and unequal allocation of work. In addition, students fall short of developing a range of soft and interpersonal skills which are critical employability skills for graduates if the assessment is not designed appropriately. In order to successfully execute group-based assessments, this research aims to develop a reliable framework for project management academics in the context of higher education.

The existing literature around group-based assessments appears to indicate some good practices for academics to undertake in group assessments. A few key patterns emerged from the initial literature review such as the frequency and timing of the assessments, group composition, and self and peer evaluations of group members. These are widely researched constructs in group assessment literature.

Academics should consider group-based assessments in a whole-of-course approach as too many group assessments in a course may have detrimental impacts on students' learning [9]. The weightage and frequency of the assessment is also an aspect which should be considered. If students are to develop soft skills through group-based assessments, they should be provided with proper training on working in a group. The significance of providing students with training is well stated in the extant literature [14].

The group formation process is an indispensable design feature of group-based assessments. Groups formed by students do not seem to experience an improved learning experience. Teacher-selected groups perform better than student-selected groups however, students prefer self-selected groups and academics tend to form groups randomly. The preliminary literature review did not establish an answer for group size; however, smaller group size was preferable.

Self and peer evaluation of group members were widely researched in the literature. Self and peer evaluations propose a wide range of benefits to students such as developing judgmental skills, critical thinking and transferable skills. In addition, self and peer assessments practices help identify social loafers in the groups. However, some students may not be able to critically evaluate peers and exhibit biased behaviour in feedback.

Some design features of group-based assessments were evident in the literature; however, the pedagogical practices seem to be far from settled. The extant literature has gone some way towards providing some indecisive findings to the discussed design aspects of group-based assessments. Hence, a thorough investigation through a SLR is required in order to explore the best practices of group-based assessments in the context of higher education. It is expected to add to the dearth of research. Once best practices are identified through a SLR, they will be contextualised within project management courses in order to be using the emerged framework to develop employability skills of project management graduates.

\section{Expected Outcomes}

It is expected that this research will contribute to the effective design of group-based assessments in higher education. The research will help identify the design features of group-based assessments and their effective pedagogical approaches. Not only will the research propose a worthwhile framework for designing group-based assessments, but it will also validate the proposed framework considering project management academics' opinions. Project management academics will be providing their opinion about the best practices derived from the SLR. Then, the proposed framework will be validated through focus groups discussion. As the process of developing the framework will be going through two stages of verification, the final framework would seem to be robust, rigorous and reliable.

It is hoped that the outcomes of this research would eventually address the contention in opinion among academics about administering group-based assessments. It is believed that the outcomes of this research will assist academics in higher education to design and operate group-based assessments systematically based on the best practices. If groupbased assessments are designed appropriately, not only can project management graduates develop employers' demanded competencies, but they can also be even more employable in the labour market.

\section{Conclusion}

Employers in the project management industry are often discontented claiming graduates are not ready for the challenges of a dynamic project environment and that they lack a range of soft skills in their skill sets. In order to address this predicament, the research will examine how group-based assessments in higher education can be designed in a systematic way in order to cultivate soft and technical skills in PM graduates.

The research aims to develop a reliable framework for designing and administering group-based assessments in PM higher education which adds to the dearth of the literature. It is anticipated that academics 
and educators will benefit from this research as the identified best practices will guide them to design group-based assessments. One can expect that the final outcome will include industry partners being able to recruit PM graduates with the demanded attributes in their skillset.

\section{Reference}

[1] A.E. Postlethwait, "Group projects in social work education: The influence of group characteristics and moderators on undergraduate student outcomes", Journal of Teaching in Social Work, 2016, pp. 256-274.

[2] A. Kamrul, M. Ho, and S. Khan, "Recruiting project managers: A comparative analysis of competencies and recruitment signals from job advertisements", Project Management Journal, 2013, pp. 36-54.

[3] A. Naomi, C.J. Woodley, W. Despina and M. Winchester, "Exploring academics' approaches to managing team assessment", International Journal of Educational Management, 2016, pp. 1150-1162.

[4] A. Praveen, and C.L. O'Brien, "Social loafing on group projects: Structural antecedents and effect on student satisfaction", Journal of Marketing Education, 2008, pp. 255-264.

[5] C. José-Rodrigo, and A. Piki, "Facilitating project management education through groups as systems", International Journal of Project Management, 2012, 83-93.

[6] D.W. Martin. "Groupwork as a form of assessment: Common problems and recommended solutions", Higher Education, 2009, 563-584.

[7] F. Johan, K.F. Frykedal, and E.H. Chiriac, "Group Work Assessment: Assessing Social Skills at Group Level", Small Group Research, 2020, pp. 87-124.

[8] F. Mark, and J. McKenzie, "SPARK, a confidential web-based template for self and peer assessment of student teamwork: benefits of evaluating across different subjects", British Journal of Educational Technology, 2002, pp. 551-569.

[9] G. Diane, R. Sally, and T. Elizabeth, "Grouped out? Undergraduates' default strategies for participating in multiple small groups", The Journal of General Education, 2006, pp. 81-102.
[10] H. Mark, and R. Land. "Assigning students in group work projects. Can we do better than random?", Innovations in Education and Training International, 2000, pp.17-22.

[11] H. Sandy, and P. Fred, "Instructor-assigned and student-selected groups: A view from inside", Issues in Accounting Education, 2010, pp. 15-33.

[12] L. Dragan, L. Bojana, D. Alesksandar, and M. Maric, "A novel metaheuristic approach for collaborative learning group formation", Journal of Computer Assisted Learning, 2018, pp. 907-916.

[13] R. Linda, A. Girardi, and C. Whitsed, "A systematic literature review of teamwork pedagogy in higher education", Small Group Research, 2016, pp. 619-664.

[14] R. Tammy L., and J.E. Mathieu, "Evaluating an individually self-administered generic teamwork skills training program across time and levels", Small Group Research, 2007, pp. 532-555.

[15] S. Keele, Guidelines for performing systematic literature reaviews in software engineering. Vol. 5. Technical report, Ver. 2.3 EBSE Technical Report. EBSE, 2007.

[16] S. Lisa M., and B.E. Valle, "Social constructivist teaching strategies in the small group classroom", Small Group Research, 2013, pp. 395-411.

[17] S. Mary, K.F. Wilson, and K.S. McKenzie, "Evaluating the quality of peer and self evaluations as measures of student contributions to group projects", Higher Education Research and Development, 2019, pp. 1061-1074.

[18] S. Samer, and R.J. Tumpa, "A systematic approach to group-based assessment in project management education: CQUniversity case study", 43RD AUBEA, 2019, pp. 207222.

[19] Willig, C., and Wendy, S.R., eds. The SAGE handbook of qualitative research in psychology. Sage, 2017.

[20] Y.G. Sahin, "A team building model for software engineering courses term projects", Computers and Education, 2011, pp. 916-922. 